Die Zukunft der Nachhaltigkeitsberichterstattung III und IV/IV

\title{
Glaubwürdigkeit können nur die Unternehmen selbst herstellen
}

\author{
Steht es Unternehmen heute noch frei, Nachhaltigkeitsinforma- \\ tionen zu veröffentlichen? Mitnichten, sagt Dieter W. Horst in \\ unserem letzten Teil der Serie zum Status quo sowie zu mög- \\ lichen Zukunftsszenarien der Nachhaltigkeitsberichterstattung. \\ Den Deutschen Nachhaltigkeitskodex stellt Yvonne Zwick vom \\ Rat für Nachhaltige Entwicklung vor. \\ Interview von Jana Gebauer
}

\begin{abstract}
Ö kologisches Wirtschaften: Aktuell werden sehr lebhaft Debatten zur verpflichtenden Nachhaltigkeitsberichter stattung wie auch zur Integration von Nachhaltigkeits- und Finanzberichterstattung geführt. Was sagt die Prüferzunft dazu, Herr Horst?
\end{abstract}

Dieter W. Horst: Ich weiß gar nicht, wer das Gerücht aufgebracht hat, dass die Berichterstattung zur Nachhaltigkeit noch freiwillig wäre. In Deutschland müssen große Kapitalgesellschaften sowie Konzerne im Lagebericht neben den finanziellen auch bedeutsame nichtfinanzielle Leistungsindikatoren darstellen. Gemäß Handelsgesetzbuch sind bei der Analyse von Geschäftsergebnis und -tätigkeit nichtfinanzielle Leistungsindikatoren, zum Beispiel zu Umwelt- oder Arbeitnehmerbelangen, zu berücksichtigen, „soweit sie für das Verständnis des Geschäftsverlaufs oder der Lage von Bedeutung sind“. Das Gleiche gilt für Mutterunternehmen bei der Erstellung eines Konzernlageberichts. Die nächste, noch freiwillige Stufe „Integrierte Berichterstattung" ist dann zugegebenermaßen mehr, als nur Schlüsselkennzahlen zur Nachhaltigkeit in den Lagebericht zu packen. Integriertes Berichten braucht integriertes Denken. Das soll nach Wunsch der EU-Kommission verpflichtend werden - siehe ihre 2011 veröffentlichte Strategie für die soziale Verantwortung der Unternehmen.

ÖkologischesWirtschaften: Was können Instrumente wie der Deutsche Nachhaltig- keitskodex (DNK) vom Rat für Nachhaltige Entwicklung (RNE) zur Diskussion beitragen? Welche Position nimmt der Rat ein, Frau Zwick?

Yvonne Zwick: Der Nachhaltigkeitsrat will Marktrelevanz für das Thema Nachhaltigkeit erreichen, ist weder für verpflichtende Berichterstattung noch den komplett freiwilligen Ansatz. Zum einen trägt die Verpflichtung an sich nicht unbedingt zu einer Anhebung der Berichtsqualität oder Nützlichkeit der Informationen bei. Zum anderen glaube ich auch nicht an den komplett freiwilligen Ansatz, unter anderem weil ich beim IÖW/ future-Ranking der Nachhaltigkeitsberichte gelernt habe, dass Freiwilligkeit der Glaubwürdigkeit schadet. Nachhaltigkeitsberichte, die vor allem vom Marketing für's Marketing geschrieben werden, haben nur begrenzten Nutzwert. Diese Lücke will der Nachhaltigkeitsrat mit dem Deutschen Nachhaltigkeitskodex schließen. Mit den standardisierten Anforderungen, die sich in zwanzig Kriterien und ausgewählte Indikatoren aufschlüsseln, schaffen wir einen Vergleichs rahmen, der zugleich eine Beschreibung des Prozesshaften von Nachhaltigkeit zulässt. Der Comply-or-Explain-Ansatz lässt $\mathrm{zu}$, dass man, wo man nicht berichten kann oder will, mit einer stichhaltigen Begründung die Anforderungen des Deutschen Nachhaltigkeitskodex erfüllt. Das ist das Wesen eines Transparenzstandards. Ziel ist, dass die Qualität der Informationen über den Markt bewertet wird, das heißt durch die Nutzer(innen) der Informationen. In unserer Zielrichtung sind das vor allem Kapitalmarktakteure, aber auch Kund(inn)en sowie Nichtregierungsorganisationen. Wenn wir es schaffen, Nachhaltigkeit zu einem marktrelevanten Thema zu machen, das Geldflüsse lenkt, haben wir eine höhere Verbindlichkeit, als es eine gesetzliche Regelung oder der rein freiwillige Ansatz erreichen kann. Seien wir doch mal ehrlich: Letzten Endes interessiert doch alle nur, wohin das Geld fließt, wo Geld verdient wird und was wirtschaftlichen Erfolg ausmacht - auch die Kleinanleger(innen) und Bankangestellten.

Horst: „Der Finanzmarkt“ will integrierte Schlüsselkennzahlen zur Nachhaltigkeit, um seine Analysen abzurunden, und zwar fokussiert auf die Schlüsselkennzahlen, die Unternehmen tatsächlich zur Steuerung ihrer Nachhaltigkeitsleistung verwenden. Materialität heißt das Zauberwort, nicht akribisches Abarbeiten irgendwelcher Checklisten von Berichtsstandards. Und dabei sprechen wir immer mehr von einer steigenden Anzahl von Mainstream-Investoren und immer weniger von den ethisch orientierten Anleger(inne)n. Es geht um professionelle, sprich um- und weitsichtige, nachhaltige Vermögensverwaltung, die Chancen und Risiken des globalen Wandels in die Anlageentscheidung einbezieht. Und dazu braucht es hochqualitative und geschäftsrelevante Nachhaltigkeitsdaten - so früh und so verknüpft mit den Finanzdaten, wie nur irgend möglich.

ÖkologischesWirtschaften: Was macht eigentlich Zweck und Wirkung der Nachhaltigkeitsberichterstattung aus - in das Unternehmen hinein, in Richtung seiner Partner und der breiten Öffentlichkeit? Kann sie Nachhaltigkeitsorientierung und integriertes Denken voranbringen?

Zwick: Ich glaube, das kann sie, aber nur, wenn sie aus der Nische kommt, raus aus den CSR- und Nachhaltigkeitsabteilungen und rein in den unternehmensinternen Dialog. Entscheidungsrelevant ist Nachhaltigkeit nicht nur in 
Forschung und Entwicklung und im Marketing, sondern genauso in Personalabteilungen, der Finanzkommunikation und im Einkauf. Ohne Netzwerkdenken lässt sich kein Unternehmen leiten. Die Führungskräfte von morgen sind darauf angewiesen, dass heute der Boden für ihr Verständnis und Denken in Netzwerken bereitet wird. Ob das geschieht, ist einer der Prüfpunkte, die uns beim Nachhaltigkeitskodex interessieren. Ich denke, da liegt eines der entscheidenden Differenzierungsmerkmale zur bestehenden Nachhaltigkeitsberichterstattung, die immer eher Vergangenheit betrachtet und den Status quo abbildet.

Horst: Nachhaltigkeit ist die Fähigkeit zum kollaborativen Denken und Handeln. Ob es um heute noch kaum denkbare Formen der Zusammenarbeit mit Lieferanten entlang der Wertschöpfungskette geht, um die Produktneuentwicklung gemeinsam mit der Kundschaft oder um komplexe Stoffkreisläufe. Auch wenn Technologie für Nachhaltigkeit bedeutsam ist, wichtiger sind die sozialen Kompetenzen und der menschliche Faktor für die "Große Transformation“. Aber Kollaboration braucht hochwertige Information als Grundlage von Vertrauen, Verständigung und abgestimmtem Handeln - und hochwertig heißt richtig, rechtzeitig, vollständig und verständlich.

ÖkologischesWirtschaften: Ist Nachhaltigkeitsberichterstattung, wie sie heute praktiziert wird, ausreichend richtig, rechtzeitig, vollständig und verständlich? Wenn nicht: Welcher Weiterentwicklungen bedarf es?

Horst: Da gibt es heute schon noch Optimierungs- und Investitionsbedarf. Systeme und Prozesse zur Datenerhebung müssen sich der Qualität der Systeme und Prozesse des Finanz- und Rechnungswesens annähern. Das wäre der Entwicklungsschritt zur Nachhaltigkeitsberichterstattung 2.0. Sehen Sie das anders, Frau Zwick?

Zwick: Ganz ehrlich? Ich glaube, dass die Nachhaltigkeitsberichterstattung die besten Zeiten schon hinter sich hat. Diese Burg lässt sich nicht halten. Mein Bild der Nachhaltigkeitsberichterstattung der Zukunft ist eines, in dem die wesentlichen Kennzahlen kontinuierlich erhoben und zu bestimmten Stichtagen in unterschiedlichen Publikationsformaten aufbereitet werden - je nach Zielgruppe. Das kann im Geschäftsbericht sein, entsprechend der Anforderungen des Kapitalmarktes, oder der zweijährliche Nachhaltigkeitsbericht, der sich an die allgemeine Öffentlichkeit richtet, oder die Entsprechenserklärung zum Nachhaltigkeitskodex mit jährlichem Update oder auch kundenorientierte Formate.

ÖkologischesWirtschaften: Bringen soziale Medien neuen Schwung - und Sinn - in die Nachhaltigkeitsberichterstattung? Und ist es hilfreich, wenn Produktund Unternehmensinformationen zusammengefasst werden, wie es bei Strichcode-Scannern und Nachhaltigkeitsampeln passiert?

Zwick: Unbedingt. Besonders wenn man an die Interaktion mit der Gesellschaft denkt oder die Information, die Kund(inn)en zum Kauf bewegen soll. Grüne Apps auf Smartphones sind gerade bei jüngeren Zielgruppen beliebt und werden an Relevanz noch gewinnen. Unternehmen kommen massiv unter Druck, auch wenn ihnen die Vermischung von Produkt- und Unternehmensinformationen nicht so gefällt. Ihnen bleibt kaum etwas anderes übrig, als in den Dialog einzutreten und selbst konstruktiv dafür zu sorgen, dass Nachhaltigkeitsampeln und ähnliches in angemessenen Abständen an die Leistungsverbesserung angepasst werden.

ÖkologischesWirtschaften: Wer kann eigentlich die Glaubwürdigkeit sicherstellen - und sehen hier alle Stakeholder ein und dieselbe Akteursgruppe in der Pflicht?

Zwick: Tja, Quizfrage. Herr Horst, das ist eindeutig Ihr Gebiet!

Horst: Wieso mein Gebiet? Glaubwürdigkeit können nur die Unternehmen selbst herstellen. Ausgangsbasis ist Information. Sie liegt der Zielfindung, der Maßnahmenplanung und der Umsetzungsüberwachung zugrunde. Je exakter sie ermittelt und je stringenter sie im Unternehmen übermittelt wird, desto besser. Wenn es um die Gestaltung oder Bewertung ausgefeilter Managementstrukturen und -prozesse, treffsicherer interner Kontrollsysteme oder eines schlagkräftigen Monitorings geht, da halte ich Prüferkompetenz für die optimale Wahl. Auch wenn es darum geht zu ermitteln, ob richtig berichtet wurde. Die Frage aber, ob das Richtige berichtet wurde, ist ein wichtiger Teil der Glaubwürdigkeit und muss von den Stakeholdern selbst beantwortet werden.

ÖkologischesWirtschaften: Frau Zwick, Herr Horst, ich danke Ihnen vielmals für das Gespräch.

\section{AUTOREN + KONTAKT}

Yvonne Zwick ist wissenschaftliche Referentin in der Geschäftsstelle des Rates für Nachhaltige Entwicklung und verantwortlich für die Themen nachhaltiger Konsum, nachhaltiges Wirtschaften und den Deutschen Nachhaltigkeitskodex.

Yvonne Zwick

Rat für Nachhaltige Entwicklung, c/o GIZPotsdamer Platz 10

10785 Berlin, Tel.: +49 30408190127 , www.nachhaltiger-warenkorb.de

Dieter W. Horst arbeitet bei dem Prüfungs- und Beratungsunternehmen PricewaterhouseCoopers und ist dort für Sustainable Finance zuständig.

Dieter W. Horst PricewaterhouseCoopers, Sustainability Services Friedrich-Ebert-Anlage 35-37, 60327 Frankfurt am Main, Tel.: +49 69 9585-1397,

E-Mail: dieter.w.horst@de.pwc.com

Jana Gebauer ist Mitarbeiterin am Institut für ökologische Wirtschaftsforschung. 
Copyright (C) 2013, IÖW und oekom Verlag. Die Nutzung des Artikels ist Abonnenten von Ökologisches Wirtschaften vorbehalten. Nachdruck und Vervielfältigung des Artikels einschließlich Speicherung und Nutzung auf optischen und elektronischen Datenträgern nur mit Zustimmung der Redaktion von Ökologisches Wirtschaften (http://www.oekologischeswirtschaften.de). 